\title{
Techno-economic feasibility analysis of Internet protocols: Framework and tools
}

\author{
Tapio Levä (corresponding author) \\ Aalto University, Department of Communications and Networking \\ P.O. Box 13000, 00076 AALTO, Finland \\ $+358505710073$ \\ tapio.leva@aalto.fi
}

Henna Suomi

Aalto University, Department of Communications and Networking P.O. Box 13000, 00076 AALTO, Finland

henna.suomi@aalto.fi

Accepted manuscript. Please cite this article as:

Tapio Levä, Henna Suomi (2013). Techno-economic feasibility analysis of Internet protocols: Framework and tools. Computer Standards \& Interfaces, vol. 36, no. 1, pp. 76-88, DOI: 10.1016/j.csi.2013.07.011.

\begin{abstract}
A multitude of Internet protocols are developed in the Internet Engineering Task Force to solve the challenges with the existing protocols and to fulfill the requirements of emerging application areas. However, most of them fail to achieve their goals due to limited adoption. A significant reason for non-adoption seems to be that the potential adopters' incentives for adoption are not understood and taken into account during the protocol development. This paper addresses this problem by developing a conceptual framework for analyzing the techno-economic feasibility of Internet protocols already during their development. The framework is based on the experiences collected during several protocol case studies and an extensive literature review. It focuses on analyzing the economic incentives of the relevant stakeholders and also takes into account the deployment environment including the competing solutions. The framework is accompanied by a research method toolbox that introduces practical tools for applying the framework. Finally, the application of the framework is demonstrated with Multipath TCP case study. The usage of the suggested framework can help protocol developers to identify the potential deployment challenges and opportunities of emerging protocols and thus increase the likelihood of adoption. Moreover, potential adopters can use the framework as a supporting tool for making adoption decisions.
\end{abstract}

Keywords: Techno-economic analysis, protocol adoption, diffusion, methodology, Multipath TCP 


\section{Introduction}

The Internet standardization community is very active in producing new Internet protocols as a response to the challenges with the existing protocols and the requirements of emerging application areas. For example, the Internet Engineering Task Force (IETF) has 127 active working groups that produced 337 working documents called as Request for Comments (RFC) during 2012 alone [1]. However, most of the new protocols never end up in use in the originally envisioned scale and scope [2]. Several reasons have been raised for explaining the lack of adoption, such as middleboxes blocking the traffic [2], difficulties in achieving critical mass [3], and lack of authorities that could mandate adoption [4]. The problems follow from the protocols being technically non-deployable or not providing sufficient economic incentives for the relevant stakeholders to implement, install, or adopt the protocol. In other words, the protocols are techno-economically infeasible.

One reason for the feasibility problems is that the protocol developers have insufficient understanding of the stakeholders' economic incentives, and thus end up designing protocols based on false or inaccurate assumptions. The IETF community has addressed this problem by identifying design principles [5,6] and protocol success factors [7] that should facilitate adoption. However, the general design principles are not sufficient to guarantee the feasibility of a protocol since they neglect the special characteristics of each protocol and mainly cover technical deployment issues leaving aside the economic perspective.

Nevertheless, the understanding of the economic factors affecting the feasibility of Internet protocols has increased gradually in the recent years as the theories and methods of innovation diffusion research have been applied in Internet protocol case studies, such as the studies of DNSSEC [3] and IPv6 [8,9]. Even though these studies suggest interesting deployment strategies and provide useful insights to the developers of future protocols, their usefulness remains limited due to two reasons. Firstly, these studies have been conducted separately from the protocol development after the protocol has already been standardized. Thus, the identified feasibility challenges cannot be taken into account in the protocol design. Secondly, they focus only on the feasibility for the potential end users of the protocol, although the success of the protocol also depends on other stakeholders, such as operating system (OS) vendors implementing and including the protocol in their operating systems.

To overcome the shortcomings of generic design principles and retrospective protocol case studies, this paper suggests an accessible and flexible framework for studying the feasibility of Internet protocols during their development. The framework is essentially a description of a systematic process for identifying the deployment challenges and suggesting strategic actions to improve feasibility. The perspective of the framework is techno-economic, as it aims to translate the technical design and protocol features into actual costs and benefits for the stakeholders. As such, it is targeted to protocol developers with limited knowledge of economics. The framework provides a set of questions grouped into different steps and is accompanied with a collection of tools that can be applied with the framework.

The framework is based on the experiences from multiple protocol case studies [10-19], including Multipath TCP (MPTCP), Mobile IP (MIP), Host Identity Protocol (HIP), 
Constrained Application Protocol (CoAP) and Locator/Identifier Separation Protocol (LISP). In addition, a literature review has been conducted to acquire a comprehensive understanding of factors that contribute to the protocol adoption. Compared to the earlier retrospective case studies that focus on the feasibility for the potential adopters of the protocol, the framework takes a wider perspective by covering also the other critical steps in protocol deployment. Protocol deployment is defined as a process during which a protocol is advanced from the first specification into actual use on the Internet. The process includes steps such as implementation, installation, and adoption of the protocol and the related technical components. This extension is relevant since the protocol deployment needs to be feasible not only to the adopters, such as the end users of the protocol, but also to the other stakeholders participating in protocol deployment.

The rest of the paper is structured as follows. Section 2 introduces the reader to the innovation and protocol diffusion studies that create the basis for the framework. The framework is then described in Section 3. Section 4 introduces a multitude of analysis tools that can be used to answer the questions posed by Section 3. Then, Section 5 demonstrates the usage of the framework with the Multipath TCP (MPTCP) case study. Finally, Section 6 discusses the applicability and limitations of the framework.

\section{Background and related work}

Diffusion of innovations has already long inspired researchers of many disciplines. The literature regarding diffusion and adoption of innovations is vast and covers both single and multi-disciplinary perspectives. The techno-economic analysis of feasibility coincides with the diffusion studies, since the feasibility of an innovation defines the diffusion rate. This section gives an overview of the existing innovation diffusion literature that creates the basis for the framework.

\subsection{Innovation diffusion studies}

Diffusion of innovations research dates back to the early $20^{\text {th }}$ century but it started to gain more momentum in the 1960s when Everett M. Rogers published his generalized theory of diffusion of innovations (DOI) aggregating the observations made on earlier studies. In his influential book, Rogers [20] describes five main attributes of an innovation, which affect the potential adopters' decision-making: relative advantage, compatibility, complexity, trialability and observability. These factors frequently appear also in the more recent studies, sometimes just with different terms (e.g., [21]). In addition to the innovation characteristics, Rogers proposes several external or social conditions that may accelerate or slow the adoption process. These vary from the extent of promotion efforts to the nature of social system which the potential adopters are involved in.

Although Rogers discusses the topic mainly from the sociological perspective, diffusion of innovations has extensively been studied in the economic literature including econometrics, marketing as well as business strategy. Economists typically see diffusion as a result of several individual adoption decisions based on the expectations of increased profits. This requires the comparison of costs and benefits of the innovation, i.e., the relative advantage 
compared to the existing technology but also to the competing solutions. This view gives a tangible idea of the cost and benefit components of adopting a new innovation but lacks an important aspect of the dynamics between stakeholders.

Studies such as [22] and [23] discuss the technology adoption in the presence of network effects, i.e., the value generated by the other adopters of the technology. The network effects can benefit the innovation adoption in several ways. For communication technologies the benefits essentially derive from the capability to communicate with other people using the same technology [24]. In addition, the growing number of adopters increases the probability for creation of complementary technologies and services that create more value to the adopters [22]. The notion of social feedback is also discussed in the marketing literature. The more adopters exist, the more they deliver information to the potential adopters in the society and the rate of adoption increases. Word-of-mouth communication can become an important booster of the adoption as the time passes [25], often referred as viral effect. On the other hand, the network effects can also hinder adoption if the benefits exceed the costs only after a certain number of adopters (known as critical mass) have taken the innovation in use. This is known as the bootstrapping problem [3].

Since the Rogers' first publication of the DOI theory, the diffusion research has become more cross-disciplinary. However, as Rogers [20] states the individual diffusion studies of the earlier era have been replaced even by an unnecessary standardization of diffusion research perspectives. Fichman and Kemerer [26] complement this view by arguing that a single predictive theory of innovation adoption and diffusion is unlikely to emerge. Therefore, studying the diffusion of Internet protocols separately is also important.

\subsection{Diffusion studies on Internet protocols}

The current layered Internet architecture still based on the original end-to end principle [27] has allowed the innovation especially at the edges of the network but fails to take into account the requirements of certain stakeholders. The challenge of the protocol diffusion derives from the fact that it requires decisions from several stakeholders and in different stages of protocol development. Each stakeholder is pursuing their own economic objectives leading to conflicting interests towards a technology and its features. Clark et al. [28] call this process a tussle and define a number of design principles for new Internet technologies to better respond to the economic requirements. Ford et al. [29] revisited those principles. A more detailed list of success factors of Internet protocols were defined in [7] which was published in the IETF as guidance for future protocol development work. Complying with these new design principles and the following characteristic guidelines in the protocol development is an important but not a sufficient condition to guarantee the success of a protocol.

Hovav et al. [30] were the first to apply the general diffusion studies on Internet standards. They defined the modes of adoption based on the usefulness of the standard's features and conduciveness of adoption environment on the Internet. They also stated that instead of seeing protocol adoption as a dichotomous outcome (between full adoption and non- 
adoption), also the existence through replacement and co-existence with the old technology are potential modes of protocol diffusion. Warma et al. [13] refined Rogers' product development process to be better applicable with Internet protocols. In addition, they stated that often end users adopt a new protocol unconsciously, which introduces a new dimension to the diffusion of innovations theory. In contrast, Levä et al. [17] took a more experimental approach when studying the factors behind the non-adoption of HIP. By interviewing different persons active in the IETF work they found out that, for example, competing solutions being developed in the IETF as well as misconceptions about a protocol within the community have affected negatively the diffusion of HIP.

Studies conducted by Joseph et al. [8] and Sen et al. [9] represent a more economic perspective on the protocol diffusion. They modeled the value of a new networking technology (IPv6) in the presence of network externalities and elaborated the role of converters as a facilitator of protocol adoption. Iannone and Levä [15] conducted a similar study on Loc/ID split protocol and elaborated the costs savings as a function of adopters in the network. These studies increase understanding of the value creation during the diffusion process, but do not reveal much on incentives and (disincentives) that lead to the actual adoption decision. An adopter-centric approach was taken by Suomi et al. [14] as well as by Warma et al. [12] who modeled the costs and benefits of MPTCP protocol to a mobile end user and a content provider, respectively.

\subsection{Methodologies for studying techno-economic feasibility}

Despite the large number of studies focusing on the factors affecting the success of new technologies, much less attention has been given on the methodologies and guidelines how to study their feasibility. Bond and Houston [31] built a framework for matching the new technologies developed within a firm to the market opportunities. Basically, the authors provide a list of challenges that managers should consider when new innovations are being developed and how these challenges could be addressed to increase their market potential. Although partly applicable to Internet protocols, the framework is primarily developed for product innovations that do not require actions from several stakeholders in the market. A white paper of Verbrugge et al. [32] suggests a methodology for techno-economic analysis of network deployment focusing on infrastructure-heavy technologies such as next generation access technologies. Consequently, this approach has strong telecom operator focus and it only concentrates on the rollout phase of new network technologies instead of the whole deployment process.

One protocol-specific effort was made by Eardley et al. [33]. They proposed a model for protocol development suggesting that, prior to the wide-scale deployment, initial easily deployable scenarios should be developed to trigger the diffusion process. Kalogiros et al. [34] developed the tussle analysis method for investigating tensions between the stakeholders. This method has been applied in practice for information-centric networking architectures [35]. Despite the effort to identify tussles on the Internet and to view the problem from the perspectives of different stakeholders, the linkage between the protocol 
development and the tussle analysis is somewhat weak because the method does not provide any concrete tools for protocol developers with varying expertise on economics.

In conclusion, to our knowledge systematic process descriptions for studying the economic feasibility of Internet protocols during their development do not exist. The framework described in the next section aims to fill this gap.

\section{Framework}

Studying the techno-economic feasibility of a protocol under development is a challenging task, which culminates in understanding whether the relevant stakeholders - including both the actual end users and other stakeholders in the value network - have incentives for participating in protocol deployment. In order to answer this question, this section describes a framework consisting of six analysis steps as illustrated in Figure 1.

Protocol specification works as the starting point for the framework. It describes the technical design of the protocol that has been produced in the protocol design process as a solution to an envisioned problem or need. The specification does not have to be finalized; already a draft version is enough to conduct useful analysis. Steps 1-4 scope the techno-economic feasibility analysis by defining the use case, technical architecture, value network and deployment environment, respectively. Then, step 5 studies the feasibility of the protocol from the perspectives of all the relevant stakeholders in order to identify the deployment challenges of the protocol. Finally, step 6 suggests ways to solve the identified challenges and to facilitate protocol deployment.

The steps are introduced in more detail in the following subsections. Each step comprises a set of questions, the answers of which form the input for the next step. Also supporting tools for answering the questions are listed for steps 2-5. These tools are introduced in more detail in Section 4. Even though the tools give structure to the analysis and help to visualize the findings, the successful usage of the framework does not necessarily require their usage. However, familiarizing oneself with the tools improves understanding of the framework.

The framework is iterative which supports its usage as a continuous tool alongside protocol development and deployment. The framework should be used multiple times to accommodate the changes made to the protocol based on the solution analysis. Because the maturity and knowledge of the protocol affect the results, the framework could also be used in different phases of protocol deployment. In addition, short and long-term analysis made at the same time may yield different results as the protocol and deployment environment are constantly evolving. 


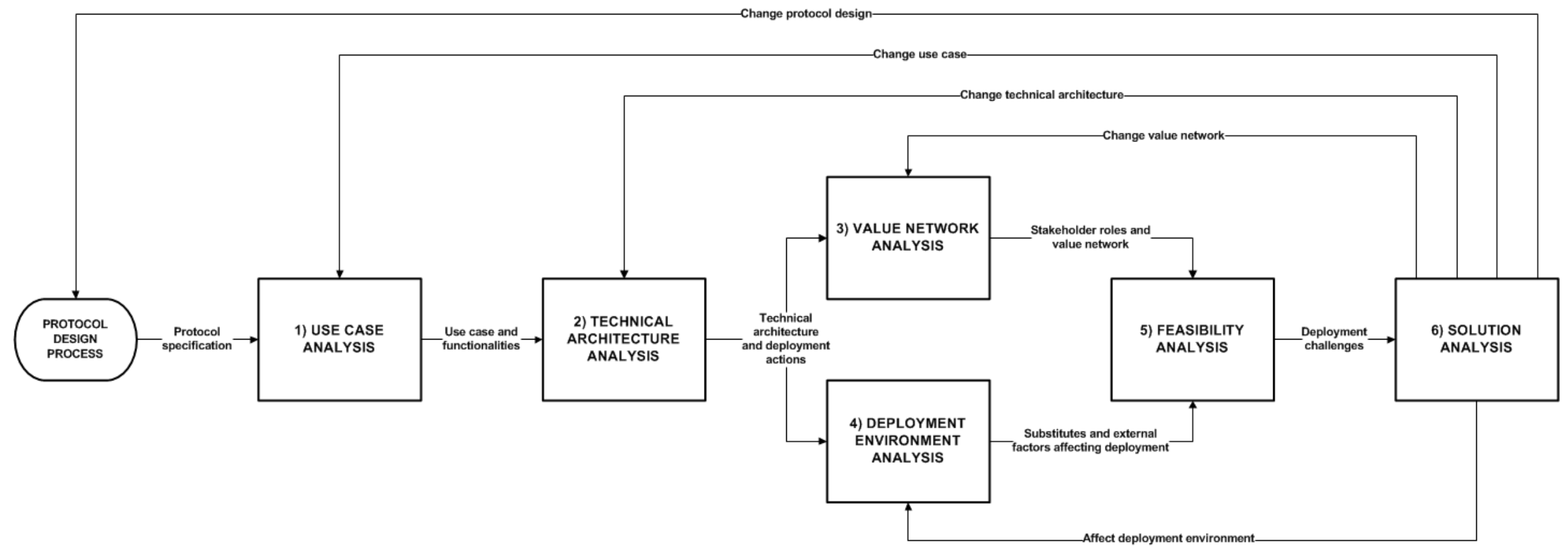

Figure 1. Framework for studying the techno-economic feasibility of Internet protocols. 


\subsection{Step 1: Use case analysis}

The use case analysis describes the purpose and functionalities of the protocol and translates them into potential use cases. Since the protocol may enable multiple use cases that differ significantly from each other in their technical architecture, value network and deployment environment (defined in steps 2-4 of the framework) ${ }^{1}$, explicit specification of the use case is required. Depending on the studied case, the definition of the use case can be narrow or wide. The description of a use case should include the selected functionality of the protocol, such as resiliency or security of a connection, which is supposed to turn into the benefits for different stakeholders in later steps. If the protocol enables multiple use cases, the complete technoeconomic feasibility analysis requires a separate analysis for each use case. Therefore, use cases should be prioritized according to their attractiveness. The most attractive use case may have the highest expected demand or least competition from other solutions, for example. When multiple use cases are analyzed, the framework can help to separate the more promising use cases from the less promising ones.

\section{Key questions:}

? What are the purpose and the functionalities of the protocol?

? What is the use case to be studied?

\subsection{Step 2: Technical architecture analysis}

The purpose of the technical architecture analysis is to describe the technical architecture and deployment actions of the use case. Deployment actions refer to all the actions that need to be taken in order to advance the protocol from the specifications into actual use on the Internet. They can relate, for example, to implementing, installing, operating, or adopting the protocol and the related technical components. Identifying these actions serves the feasibility analysis as they incur costs that the involved stakeholders have to bear if they decide to participate in protocol deployment. The incremental protocols typically have less deployment actions than the more disruptive ones.

Often the technical design and the chosen use case allow multiple technical architectures with different deployment actions. A typical example with Internet protocols is an alternative to deploy a protocol either in end-hosts or in a proxy in the middle of the communication path. Similarly, a protocol can be implemented in the OS kernel (e.g., TCP/IP), as an application layer middleware (e.g., Transport Layer Security (TLS)), or directly in applications (e.g., BitTorrent). The framework does not mandate the choice of a single deployment scenario, which allows a comparative analysis of multiple technical architectures. However, without narrowing the scope to a single technical architecture, the analysis may prove to be too demanding in the following steps.

\footnotetext{
${ }^{1}$ For example, HIP can be used as a solution to mobility management or to securing communications in virtual private networks [17].
} 


\section{Key questions and tools:}

? What is the technical architecture to be studied?

X Technical architecture modeling, flow diagram

?hat are the required deployment actions in the chosen technical architecture?

\subsection{Step 3: Value network analysis}

Value network analysis maps the deployment actions to the stakeholders responsible for taking them. This translates into identifying the relevant stakeholders and their roles in protocol deployment. In this paper, a stakeholder is defined as any group or individual who can affect or is affected by the deployment of the protocol. This covers both the stakeholders needed for advancing the protocol from the specification to the use during protocol deployment - such as implementers, installers, and adopters of the protocol - and stakeholders such as regulator that can indirectly affect the success of the protocol by either facilitating or hindering the deployment. In case the number of relevant stakeholders is high, they can be prioritized based on their power to influence deployment in order to channel the analysis effort reasonably in step 5.

Besides mapping the deployment actions to the stakeholders, the value network analysis defines how the service enabled by the protocol is provided. In practice, this means identifying how the goods and services, money or intangible benefits are exchanged between the stakeholders. This information is used in step 5 to allocate the costs and benefits to stakeholders and to analyze the incentives of each stakeholder to participate in protocol deployment.

Similarly to technical architectures, multiple value networks are typically possible. For example, a vertically integrated stakeholder might take the responsibility of all the deployment actions, or the actions could be divided horizontally among multiple stakeholders. The framework allows the comparison of multiple value networks, but focusing first on one value network and then expanding to other alternatives is recommended.

\section{Key questions and tools:}

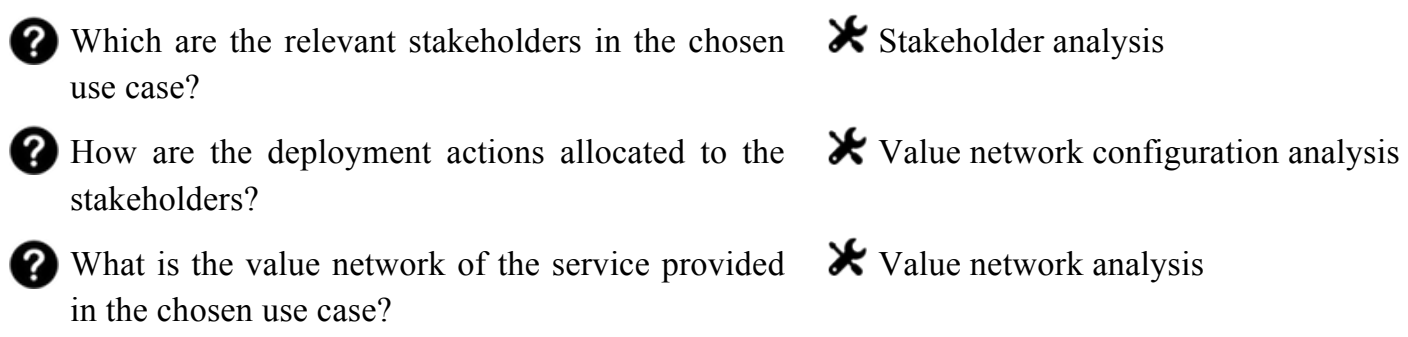

\subsection{Step 4: Deployment environment analysis}

As protocols are rarely deployed in a void, understanding the environment where the protocol is to be deployed is essential. Deployment environment analysis can be divided into the analysis of the substitutes of the protocol and the wider environmental analysis incorporating 
also the political, economic and social aspects. Analysis in this step is technology-centric, as against the stakeholder-centric analysis of step 5 .

The analysis of the substitutes starts with their identification. The substitutes of the protocol include both the incumbent solutions the protocol is aiming to replace and the other new solutions under development. Substitute solutions include all competing solutions - not only the perfect substitutes - which offer similar functionalities as the protocol in question. Even though the identification of the substitutes may seem a trivial task, it needs to be conducted carefully to avoid missing some relevant substitutes or including unnecessary ones. The use case definition helps in deciding, which solutions truly solve the same problem and can substitute the protocol under study. In addition to other protocols at the same or different layer of the protocol stack, substitutes can be, for example, application-specific solutions ${ }^{2}$. After the identification, performance and deployment status of the substitutes should be compared against the protocol under study. This provides useful input and helps in deciding which substitutes are included, when the relative advantage of the protocol is analyzed from the stakeholder-centric perspective in step 5.

The wider environmental analysis incorporating the political, economic, social and technical aspects helps to identify the external factors potentially affecting the feasibility of the protocol. For example, the prevailing regulation may limit the feasibility. The technical domain should incorporate all relevant technical factors besides identification of substitutes, such as limited battery lifetime in devices. Additionally, this step can incorporate exploring the possible evolution paths of the deployment environment in order to understand the trends that are favorable and non-favorable from the perspective of the studied protocol. This is especially important if the protocol development is in the early phase and the protocol will mature for deployment not earlier than in few years' time.

\section{Key questions and tools:}

? What are the relevant substitutes of the protocol in the use case and how do they compare against it?

? What are the relevant external factors affecting feasibility of the protocol and how can the deployment environment evolve in the future?
※ Venn diagram, comparison table, performance measurements and simulations

PEST analysis, SWOT analysis, scenario planning

\subsection{Step 5: Feasibility analysis}

After steps 1-4 have scoped the study, step 5 analyses the feasibility of the protocol by evaluating if the relevant stakeholders have incentives to participate in protocol deployment. In practice, this translates into comparing the costs and benefits of the protocol for each relevant stakeholder. Therefore, to understand the feasibility of the protocol, the analysis

\footnotetext{
${ }^{2}$ For example, mobility (i.e., allowing communication to continue when host moves between different networks) can be solved with network-layer protocols (e.g., Mobile IP, HIP), at the link layer (e.g., cellular networks), or directly by applications (e.g., email clients, web browsers) [17].
} 
needs to take into account all the relevant stakeholders identified in step 3 and the substitutes and other relevant factors identified in step 4.

The fundamental question in protocol feasibility is whether a real need for the protocol exists in the market. This is an important point of analysis since the problem that the protocol solves may not correspond to the demand of the stakeholders. Therefore, the potential adopters may not see any value in the protocol if they are happy with their current (good enough) solutions, and the perceived benefit (i.e., the willingness to pay) may be close to zero.

Assuming that the demand for the protocol exists, the minimum condition for the feasibility of the protocol is that its benefits cover its costs or, in other words, its utility is positive for all relevant stakeholders. On the positive side, the protocol may create revenues or cost savings. The costs, on the other hand, can be capital expenditures (CAPEX), such as purchasing software or hardware, or operational expenditures (OPEX), such as electricity costs or salaries of personnel. In the feasibility analysis, all the technical parameters such as interoperability or increased bandwidth can be turned into cost or benefit components.

Although the protocol would introduce positive utility for each stakeholder, it should also have relative advantage over its substitutes, including both the incumbent solutions that the protocol is aiming to replace, and the substitutes under development. The separation of studying the feasibility of a protocol, first independently of, and then in relation to, substitutes may be challenging, especially if there is an incumbent solution that the protocol is replacing. Therefore, the business case of the protocol is often studied immediately in comparison to its substitutes. ${ }^{3}$

The static, separate analysis of each stakeholder is not sufficient, because the costs and benefits of the protocol often depend dynamically on the number of adopters. Therefore, studying the existence and impact of network effects, both between similar and different types of adopters, is essential. Typical questions to ask are do the early adopters have incentives to adopt and what is the critical adopter mass after which the benefits exceed the costs. To underline the importance of network effects, the utility of the protocol can be divided into standalone utility (does not depend on other adopters) and network utility (depends on other adopters).

Estimating the values of the cost and the benefit components may be challenging, especially during the early phases of the protocol development when the level of uncertainty is high. This presents a challenge to feasibility analysis but does not prevent it. The main objective of feasibility analysis is namely the identification of the deployment challenges of the protocol, which can often be done already based on the qualitative analysis. The deployment challenges typically result from the relevant stakeholders' lack of incentives to participate in protocol deployment.

\section{Key questions and tools:}

\footnotetext{
${ }^{3}$ For example, MPTCP has often been compared to regular TCP that is the dominant incumbent protocol $[12,14]$.
} 
?

Does the protocol demonstrate positive net benefits for all the relevant stakeholders?

?

How do network effects impact on the formation of costs and benefits?

? What are the deployment challenges of the protocol?
Q Qualitative cost-benefit analysis, functional modeling, techno-economic modeling

Functional modeling, system dynamics

Combination of feasibility problems

\subsection{Step 6: Solution analysis}

After the feasibility for each stakeholder and the potential deployment challenges of the protocol have been identified, the protocol developers or other advocates should consider carefully how the challenges could be solved. Basically, five high-level solutions exist: 1) changing protocol design, 2) changing use case, 3) changing technical architecture, 4) changing value network, and 5) affecting deployment environment. The choice of the solution depends on the problem at hand, the development phase and the maturity of the protocol, and the power of the protocol advocates to impact on protocol design, technical architecture, value network, and deployment environment.

Firstly, a fundamental problem in the protocol design - such as the lack of backwards compatibility, performance issues or problems with middlebox traversal - may require changes to the protocol design. These kinds of issues should be identified as early as possible, because protocol design can be easily changed only in the early phases of the development before the protocol has been standardized.

Secondly, a protocol may not be sufficiently feasible in the selected use case due to, for example, too hard competition from substitutes or disinterest of an essential stakeholder to participate in protocol deployment. In this case, the protocol may need to be scoped again for other use cases. As a result, the framework can help to separate the promising use cases from the non-promising ones.

Thirdly, technical architecture, though heavily dependent on the protocol design, can often be changed without changing the protocol design itself. As a result, some of the problematic deployment actions can possibly be eliminated or replaced with easier actions. For example, a functionality originally implemented in a central server could be distributed to multiple locations to improve the performance of the service. Similarly, other functionality could be implemented in a proxy instead of end hosts to reduce the number of implementation locations and stakeholders involved.

Fourthly, a typical challenge with Internet protocols is the misalignment of costs and benefits, which could in some cases be solved by payments or other business agreements between the participating stakeholders. Also incentive mechanisms, such as bundling of complement products and monetary subsidies, could be used to facilitate protocol deployment in cases where the business case is not sufficiently attractive to some stakeholders. Reallocating the value network roles under the umbrella of a single actor by bundling the unprofitable deployment actions with the profitable ones is a more fundamental way to change the value network. The fewer stakeholders are involved, the less expensive coordination is required. 
This also reduces the risks related to other stakeholders' non-deployment, an extreme case being one where a single stakeholder fully controls the deployment.

Finally, affecting the deployment environment proposes yet another opportunity to increase the feasibility of the protocol. This may include actions like lobbying the politicians responsible for legal decisions in favor of the emerging protocol or creation of alliances that support the protocol. However, affecting the deployment environment is a challenging strategy for facilitating the protocol deployment and the other solutions introduced above are presumably more effective.

Before implementing the solutions, their impact should be analyzed by going through the framework again. The more fundamental the change is, the more thoroughly the analysis needs to be repeated. In general, the framework should be used iteratively as long as all the potential feasibility challenges have been solved or they are deemed to be unsolvable. The analysis should also be repeated multiple times during the protocol deployment when the knowledge of the protocol's utility and stakeholders' incentives improves.

\section{Key questions:}

\footnotetext{
? What are the potential solutions to the identified deployment challenges?

? How would the implementation of the potential solutions affect the feasibility of the protocol?
}

\section{Tools}

The framework in the previous section sets the guidelines for evaluating the feasibility of a protocol and lists potential tools that can be used in the course of the application of the framework. This section introduces these tools in more detail. Additionally, a comparison of tools is provided in order to facilitate the choice of the most suitable tool depending on the modelers' techno-economic knowledge and available resources, for example. Please note that no tools are introduced for steps 1 and 6 due to their straightforward nature and lack of suitable tools. Moreover, the set of tools is not comprehensive so the users of the framework should complement the list with other potential tools if seen applicable.

\subsection{Tools for technical architecture analysis (step 2)}

As presented in Section 3, the purpose of the technical architecture analysis is to understand what kind of deployment actions a new protocol entails and how they map into technical components. Technical architecture figure (see example in Section 5.2, Figure 4) is an effective tool for visualizing the basic infrastructure of the protocol and changes that are needed to the legacy system. Drawing the protocol stack into the same figure with the network devices that are supposed to run the new functionalities is another and more detailed approach, For example, in RFC 6182 the protocol stack of MPTCP is mapped into the Internet architecture [36]. The strength of this approach is that the model also shows the requirements for upper and lower layer protocols.

Flow diagram (or message sequence chart) is a useful tool if there is a need to describe the messaging flows between the technical architecture components. Wu et al. [37] provide an 
example of flow diagram for a handoff scheme extension to MIP protocol. However in many cases, just showing the technical interface is enough and no further communication flow modeling is required. Often, both the technical architecture and the flow diagram can be found straight from the technical specifications of the protocol or they can be drawn by using the information of the specifications complemented with discussions with the protocol developers.

\subsection{Tools for value network analysis (step 3)}

The tools for value network analysis illustrate how a network of stakeholders provides a product or a service to end users. We cover here three tools: 1) stakeholder analysis [38][39], 2) value network configuration methodology [40], and 3) Allee's value network analysis [41].

Stakeholder analysis aims for identifying the stakeholders and suggesting how managers should pay attention to each stakeholder. Stakeholder analysis typically classifies the identified stakeholders based on different attributes, such as power and interest [42], or power to influence, the legitimacy of each stakeholder's relationship with the organization, and the urgency of the stakeholder's claim on the organization [38]. For a protocol-specific study, Levä et al. [10] mapped the stakeholders of MPTCP based on their level and timescale of interest. For the purposes of the framework, the stakeholder analysis is valuable both for identifying and prioritizing the relevant stakeholders.

Value network configuration (VNC) methodology by Casey et al. [40] maps the technical architecture and the value network together by grouping activities and technical components into roles and assigning these roles to stakeholders (called as actors). The graphical notation used by the methodology is described in Figure 2. The technical architecture is visualized by connecting the technical components into each other with technical interfaces, such as protocols. The value network configuration emerges as actors take on roles and enable business interfaces, such as agreements, among each other.

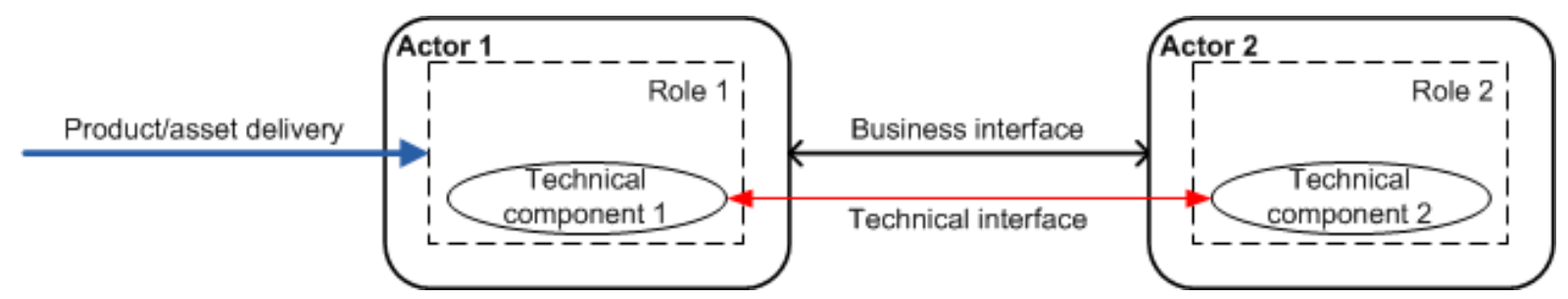

Figure 2. Notation used in the value network configuration methodology

The separation of roles from actors helps in identifying the responsibilities of each stakeholder and provides an illustrative tool for mapping the deployment actions to stakeholders. Because deployment actions incur some costs to the stakeholders, the VNC can be used to identify who bears these costs. Furthermore, the visual notation suits naturally for comparison of multiple value networks that the same technical architecture enables.

Allee's value network analysis illustrates graphically how a web of relationships generates value through complex dynamic exchanges [41]. Value exchanges are typically divided into 
different categories, such as 1) goods and services, 2) monetary benefits, and 3) intangible benefits (e.g., reputation, information). In graphical notation (see example in Section 5.3, Figure 5) different arrow types are used for the different categories and each arrow includes an explanatory text describing the exchanged value.

The value network analysis explains the business model of the provided service for each stakeholder. The value received translates into revenue, whereas the value given typically induces costs. However, the visual notation remains on a rather high level due to which each value exchange needs to be studied deeper (with the tools used) in the feasibility analysis. Some examples of using value network analysis can be found in the papers of Zhao [43], Faratin [44] and Zhang et al. [45].

\subsection{Tools for deployment environment analysis (step 4)}

Venn diagram (see example in Section 5.4, Figure 6) is a useful tool for identifying and positioning the substitutes of the studied protocol in cases where the protocol provides multiple functionalities. Each functionality is presented as a circle, the studied protocol is in the area where all the circles overlap, and the substitutes are positioned based on their functionalities. Depending on the chosen use case, some substitutes can be excluded from the further analysis if they do not enable all the required functionalities of the use case.

Comparison table is a classic tool for comparison of the substitutes covering a large number of parameters. The visualization of the table can be enhanced by standardizing the values (e.g., high/medium/low) and color coding the cells of the table based on the goodness of the values. Performance measurements and simulations of protocols, which are widely used by engineers today, are the key data sources for the quantitative protocol comparison [46].

Wider environmental analysis has traditionally been understood as a firm-centric analysis of a firm's business environment. However, the tools developed for business environment analysis can also be applied to technology-centric analysis of the deployment environment. PEST analysis is an environmental scanning framework that can be used to identify the Political, Economic, Social and Technological factors possibly affecting the protocol deployment [47]. Even though PEST can provide useful information about the environment alone, it is typically used to in conjunction with other analysis tools to ensure that all the relevant perspectives have been taken into account.

SWOT analysis (see example in Section 5.4, Table 2) is a qualitative analysis framework to evaluate the Strengths, Weaknesses, Opportunities and Threats of a project or a business venture [48] - or a protocol as used in this paper. SWOT requires a clearly defined objective, which in this case is the successful deployment of the protocol. Then the internal $(\mathrm{S}+\mathrm{W})$ and external $(\mathrm{O}+\mathrm{T})$ factors favorable and unfavorable to the objective are identified and collected into a $2 \times 2$ matrix. Authors have criticized SWOT being a mechanical tool that neither guides its appliers to prioritize the identified factors nor suggests a way to utilize the results [48]. Thus, conducting SWOT analysis alone may not be sufficient but it should be accompanied with other methods. 
Scenario planning, often accompanied with PEST analysis, is an established tool for exploring the possible evolution paths of certain industry [49]. Scenario planning builds on identifying the trends and uncertainties. Trends describe the collective knowledge of the industry and form a base for all the scenarios, whereas uncertainties differentiate the scenarios from each other. Since protocol deployment typically lasts for years, scenario planning helps to understand the evolution of the deployment environment, which may end up improving or impairing the feasibility of the protocol. Hence scenario planning is an especially useful tool during the early phases of the protocol development. Plenty of examples [50-53] of using scenario planning with emerging mobile and Internet technologies are available.

\subsection{Tools for feasibility analysis (step 5)}

Qualitative cost-benefit analysis, also known as pros and cons analysis, evaluates the feasibility of the protocol by listing the positive and negative factors of protocol deployment for each relevant stakeholder. Due to its straightforward nature, qualitative cost-benefit analysis is typically used in the beginning of feasibility analysis to get a glimpse of the potential deployment challenges and to identify the most interesting issues for more elaborate analysis.

Functional modeling is a natural extension to the qualitative cost-benefit analysis. It puts the cost and benefit components into a functional format. This creates a baseline for quantitative analysis. The basic idea behind the functional modeling methods is to create and understand functional behavior of a certain phenomenon, such as the stakeholder profit or social value of the protocol. Thereafter, numeric values of the model variables can be estimated and simulations or example calculations can be executed. To better analyze the functional behavior, model variables are typically given theoretical values often normalized between zero and one.

Essentially, functional modeling methods include utility and game theoretic modeling. In utility modeling a function models the (net) benefits of the protocol for certain stakeholder. Since network protocols are prone to network effects, a common way is to model the utility of the protocol as a function of other adopters in the networks. Joseph et al. [8] followed this approach for modeling the utility of IPv6 and Iannone and Levä [15] for Locator/ID split protocol. Game theoretic modeling tackles the problematics of decision-making. Different game theoretic models analyze the strategies of decision-makers in different contexts. The decision-making context can vary, for example, based on the degree of co-operation and timing of the decision-making between stakeholders. Tang et al. [54] modeled the profit outcomes of Internet Service Providers (ISP) in a situation where end users can balance their load between two ISPs and ISPs need to decide the amount of bandwidth they offer.

Techno-economic modeling proposes a more pragmatic approach for analyzing the profitability of a technology investment [55]. A techno-economic model essentially requires real-world (or at least close to real-world) estimations for each cost and revenue component. Especially for cost components, market data and interviews with the industry experts 
introduce a good source of information. Often, a lot of uncertainty is related to the cost and revenue estimations and, therefore, a techno-economic analysis should always incorporate a decent sensitivity analysis. To guarantee a fair comparison between the costs and the revenues of the protocol, a techno-economic model should always be created from the perspective of a certain stakeholder.

Several approaches exist for conducting techno-economic modeling. The most traditional approach is discounted cash flow (DCF) analysis. DCF is a common practice for companies to estimate the value of their investments where costs and revenue of an investment materialize at different times [56]. A study conducted by Warma et al. [12] used DCF analysis to estimate the profitability of MPTCP to a group of content providers.

Often the numerical values for cost components are fairly easy to estimate but the quantification of benefits is a more challenging task. In this case, DCF analysis becomes inapplicable. One alternative is to use total cost of ownership (TCO) analysis, which is a tool for analysing the costs of the entire lifecycle of a product or service [57]. Since it does not take a stance on the revenues of the protocol, TCO analysis is feasible only for comparing the costs of protocols having the same expected benefits. Levä et al. [18] applied the TCO analysis to the comparison between CoAP and the dominant application layer protocol, Hypertext Transfer Protocol (HTTP).

Another potential approach, which does not attempt to assign monetary values for the benefits of the investment, is called cost-effectiveness analysis (CEA) [58]. In CEA, the costs of the investment are compared to the non-monetary outcomes. This tool has traditionally been used to aid political decision-making in health care investments, but Sonntag and Suomi [19] extended its usage to Internet protocols in their recent study concerning the feasibility of MPTCP. In case of emerging protocols, the potential of CEA lies in comparing the amount of money that need to be spent to obtain an extra unit of connectivity time, bandwidth or other non-monetary benefits that the protocol is supposed to entail. Then it is up to the different stakeholder to decide whether the protocol's benefits justify investment.

System dynamics (SD) is a tool for modeling complex systems and its primary purpose is to increase understanding of underlying interactions of a certain system [59]. SD is a visual tool that can illustrate the causal relations and their type between system variables, which would be otherwise, e.g., through mathematical functions, difficult to determine. Therefore, SD is very applicable for problems related to interdependencies between stakeholders. Positive relation means that the effect of a certain variable is positively related to the cause whereas the negative relationship has an opposite effect.

System dynamics can be applied both in the qualitative and quantitative level. Qualitative modeling is able to illustrate the interactions between certain variables in the system, e.g. a factor affecting adoption decision. Quantitative format requires defining the functions of relations and initial values. Figure 3 explains the basic notation of SD modeling by illustrating how the adoption rate of a product affects the number of adopters and potential adopters in the society. 


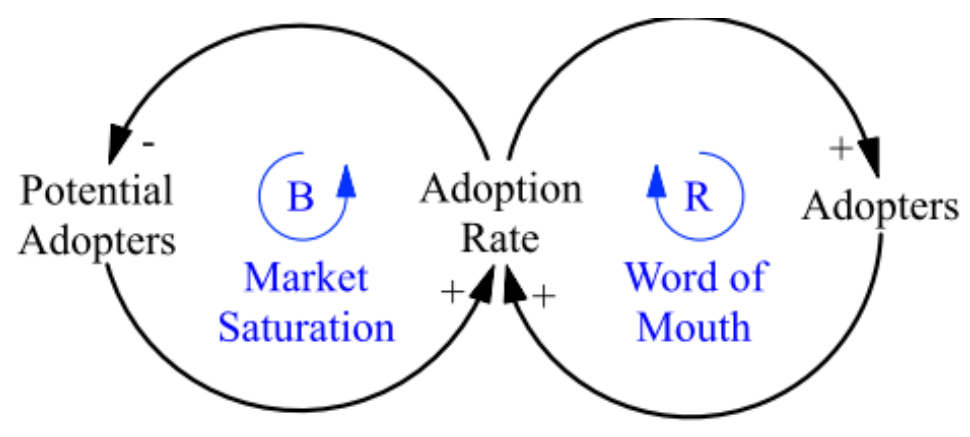

Figure 3. . An example of causal relationships in system dynamics.

In its quantitative format, SD improves understanding of the endogenous structure of the system and how it may cause non-linear outcomes, such as exponential growth rate of a technology. However, the data collection regarding future oriented problems as well as soft variables, such as mental models of people, proposes a challenge for quantitative SD modeling. Examples of using SD modeling with communication technology adoption can be found in studies conducted by Thun et al. [60], Kelic [61] and Warma et al. [13].

\subsection{Evaluation of tools}

Since several tools can be used to answer the questions posed by the framework, the choice between the tools is not always trivial. Available data, other resources and the type of the protocol under scrutiny are central issues that affect the choice. To alleviate the problem, Table 1 summarizes the potential data sources and laboriousness of the tool. In addition, the examples of protocol studies using a specific tool have been listed, because following an example facilitates the learning.

A logical approach for applying the framework is to start with the qualitative and less laborious tools moving towards quantitative and more sophisticated modeling. Basically, all the tools proposed to be used prior the feasibility analysis step are qualitative. Also in the feasibility analysis step, listing the costs and benefits and conceptualizing the interactions between stakeholders qualitatively is an efficient way to start.

Even though it is possible to get relatively far by qualitative visualization and modeling tools, the data collection should not be neglected. Simulations and measurements of network performance and traffic can provide objective data for analyzing the relative advantage of the protocol in comparison with its substitutes. To get a comprehensive view on the incentives and disincentives for deploying the emerging protocols, also the opinions of different stakeholders are crucial from early on so that the analysis does not end up presenting the modeler's subjective view. For example, questionnaires, interviews and brainstorming can provide rich data. 
Table 1. Characteristics of analysis tools

\begin{tabular}{|c|c|c|c|}
\hline \multicolumn{4}{|c|}{ Step 2 - Technical architecture analysis } \\
\hline $\begin{array}{l}\text { Technical architecture } \\
\text { modeling }\end{array}$ & Technical specifications, interviews & Low & MPTCP [36] \\
\hline Protocol stack figure & Technical specifications & Low & HIP [17] \\
\hline Flow diagram & Technical specifications & Low & MIP [37] \\
\hline \multicolumn{4}{|c|}{ Step 3 - Value network analysis } \\
\hline Stakeholder analysis & Interviews & Low & МРТCР [10] \\
\hline VNC analysis & Interviews, brainstorming & Medium & ICN [35] \\
\hline Value network analysis & Interviews & Medium & $\mathrm{ICN}[45]$ \\
\hline \multicolumn{4}{|c|}{ Step 4-Deployment environment analysis } \\
\hline Venn diagram & Technical specifications, interviews & Low & HIP [17] \\
\hline Comparison table & Simulations, performance measurements & Medium & IP mobility [46] \\
\hline $\begin{array}{l}\text { PEST, SWOT, } \\
\text { scenario planning }\end{array}$ & Interviews, brainstorming, questionnaires & Medium & P2PSIP [51] \\
\hline \multicolumn{4}{|l|}{ Step 5 - Feasibility analysis } \\
\hline $\begin{array}{l}\text { Qualitative cost-benefit } \\
\text { analysis }\end{array}$ & Interviews, questionnaires, market reports & Low & MPTCP [10] \\
\hline Functional modeling & $\begin{array}{l}\text { Interviews, market data, traffic and } \\
\text { performance measurements }\end{array}$ & High & $\begin{array}{l}\text { BGP [62], } \\
\text { IPv6 [8], } \\
\text { LISP [15], } \\
\text { MPTCP [14] }\end{array}$ \\
\hline $\begin{array}{l}\text { Techno-economic } \\
\text { modeling }\end{array}$ & $\begin{array}{l}\text { Interviews, questionnaires, market reports, } \\
\text { simulations, performance measurements }\end{array}$ & Medium/High & $\begin{array}{l}\text { MPTCP [12], } \\
\text { CoAP [18] }\end{array}$ \\
\hline System dynamics & $\begin{array}{l}\text { Interviews, market data, traffic and } \\
\text { performance measurements }\end{array}$ & High & MPTCP [13] \\
\hline
\end{tabular}




\section{Application of the framework - the case of Multipath TCP}

In this section the framework is used for analyzing the feasibility of MPTCP, which is being developed by the MPTCP working group of the IETF. RFC 6182 [36] defines the architectural guidelines of MPTCP whereas RFC 6824 [63] contains the technical specification of the protocol. These documents work as a starting point for our analysis.

The purpose of this section is not to conduct in-depth analysis but to demonstrate the usage of the framework. Therefore, we refer heavily to our earlier MPTCP studies [10-14] that have covered different steps of the framework. Furthermore, the framework has been partially applied to HIP, CoAP and MIP in other recent papers [16-18].

\subsection{Use case analysis}

Purpose and functionalities: The ability to dynamically select between different paths on per-packet basis allows optimization over several criteria, such as latency, throughput, communication cost, and energy consumption. MPTCP is one protocol that exploits multiple paths between hosts and aims for better utilization of network resources. By balancing the traffic throughout the network, the overall throughput and resiliency can be improved without the need to increase capacity. MPTCP enables seamless mobility between different access networks to improve resiliency of the connection, and simultaneous usage of multiple paths to improve throughput.

Use case: The functionalities of MPTCP are potentially useful in multiple use cases including, e.g., client-server based content services, peer-to-peer file sharing and data center networking. In this paper, we focus on the first one where MPTCP is used to improve the performance and end-user experience of client-server based content services, such as YouTube. There, consumers (client-side) use multihomed (e.g., 3G+WLAN) mobile devices to access content provided by multihomed content providers (server-side).

\subsection{Technical architecture analysis}

Technical architecture: The technical architecture under study is illustrated in Figure 4. Both client and server hosts are multihomed, even though MPTCP requires only one end to be multihomed. The routers in the middle forward packets based on the IP address of each interface, which deviates the paths between the client and the server. The original specification of MPTCP is transparent to applications but an extended application programming interface (API) would give the application more control over MPTCP behavior [64]. This analysis assumes the legacy API and modifications only in the transport layer, which are indicated with gray background color in Figure 4. Other technical architecture (and value network) alternatives for providing the multihomed access to consumers are introduced in the paper of Levä et al. [10]. 


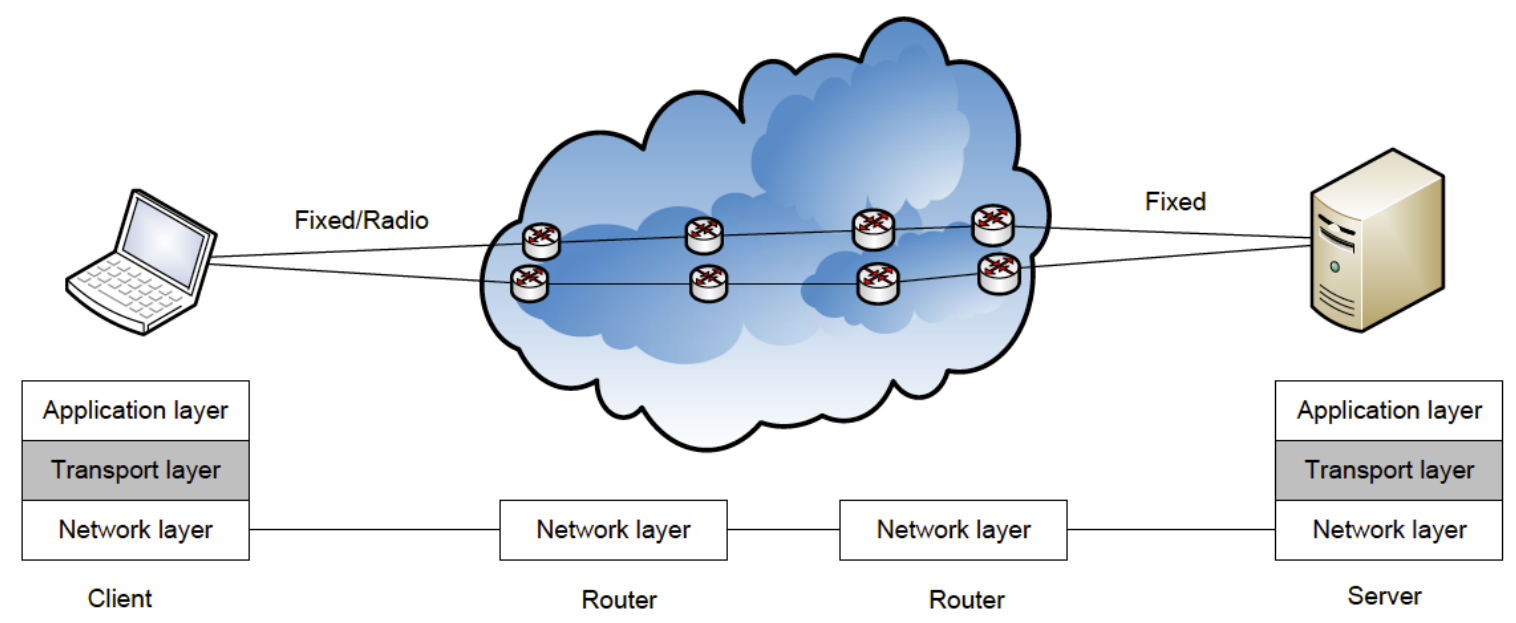

Figure 4. Technical architecture of the chosen use case.

Deployment actions: Deployment actions of MPTCP identified in the paper of Kostopoulos et al. [11] include:

- Implement MPTCP and other software changes for the operating systems of the client and server devices.

- Acquire multihoming capability on the client and the server sides.

- Install MPTCP to devices.

- $\quad$ Take MPTCP into use.

\subsection{Value network analysis}

The deployment process of MPTCP requires actions from several actors in the market. Levä et al. [10] and Kostopoulos et al. [11] identify and describe the relevant stakeholders and their deployment actions:

- OS vendors or application providers implement MPTCP in operating systems or applications for use on end systems and decide if MPTCP is enabled by default in the shipping configuration. The following analysis focuses on the OS implementation.

- Device vendors decide, which OS version is pre-installed in the devices. The significance of device vendors depends on the importance of device acquisition as a diffusion channel for operating systems and the level of device vendors' selectiveness for the OS version. Since device vendors typically provide the newest available version of the OS in their devices, they are not very critical for MPTCP deployment and are left out from the feasibility analysis.

- Consumers are end users of MPTCP that use content services provided by content providers. They own mobile devices, acquire multihoming capability and possibly install and take MPTCP into use. 
- Content providers are end users of MPTCP that provide content services to consumers. They own the content servers and install the MPTCP into them. They also acquire multihoming capability.

- ISPs provide connectivity for multihoming. Active participation from ISPs is not required in order to deploy MPTCP because end users can acquire multihoming support by making separate agreements with multiple ISPs. However, ISPs may support or hinder deployment by promoting multihoming or blocking MPTCP traffic, respectively.

Consequently, OS and device vendors, consumers and content providers are stakeholders who can directly affect the deployment whereas ISPs are affected by the deployment of MTPCP when it is taken into use on the Internet. The value network that the stakeholders constitute is illustrated in Figure 5. It visualizes the allocation of deployment actions to the stakeholders and explains the high-level business model of each actor. Typically, the provider of a product or a service receives money from its customer but other kind of value exchange may exist. For example, the consumer does not necessarily need to pay money directly to the content provider if he exposes his usage information to be used for advertising purposes. This is illustrated with dotted line in Figure 5. Continuous and dashed lines illustrate product/service and money exchange, respectively.

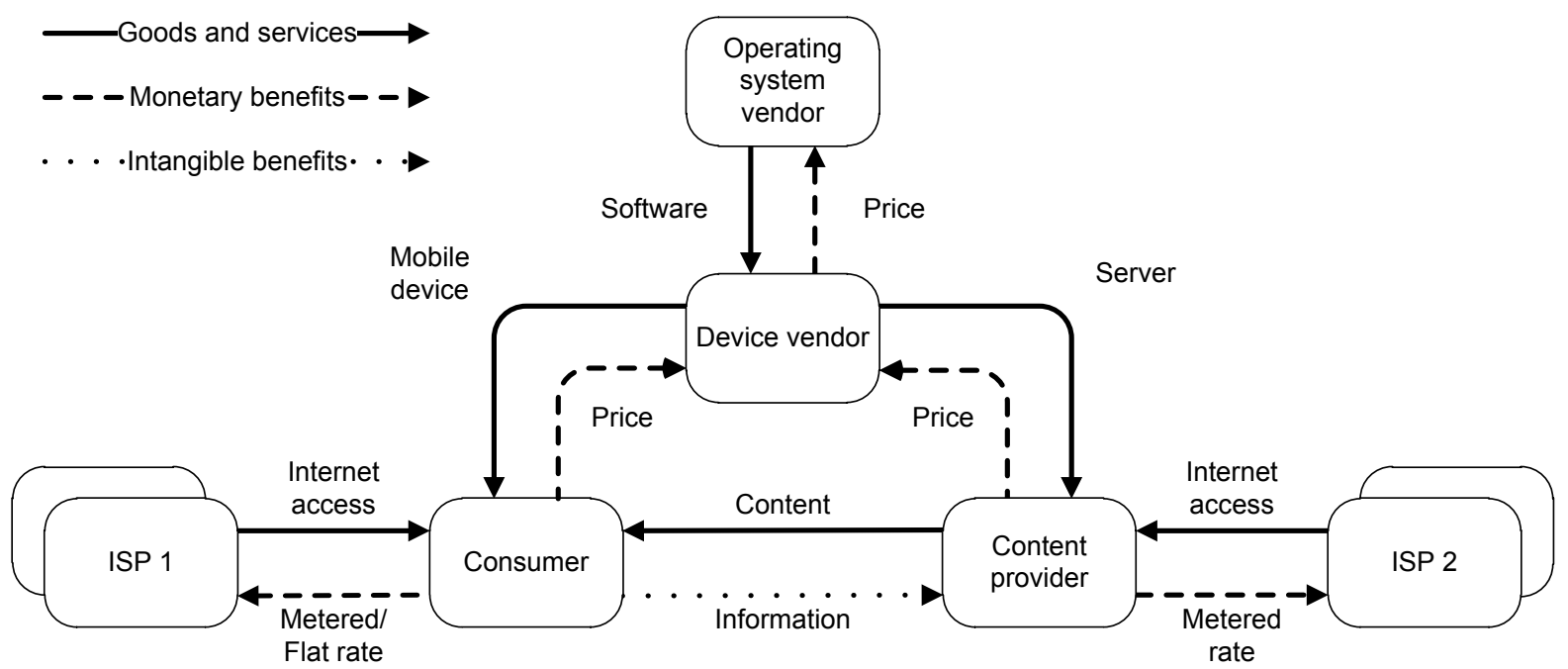

Figure 5. . Value network of the chosen use case

\subsection{Deployment environment analysis}

Substitutes: Most importantly, Multipath TCP is positioned as an extension to TCP, which is the most utilized transport protocol on the Internet. Therefore, the minimum requirement for MPTCP is to have relative advantage over TCP. The single-path TCP as such does not support any of the specific functionalities of MPTCP, but some applications open concurrent TCP connections to increase the bandwidth of the content transfer. 
The other potential substitutes for MPTCP are grouped in Figure 6 based on the functionalities they provide. The diagram includes a number of substitutes that could provide similar functionalities as MPTCP, namely mobility and multipath transfer. In addition, substitutes that would provide only one of these functionalities are also listed. Some of the substitutes such as Session Initiation Protocol (SIP), Multipath Real-time Transport Protocol (MPRTP) and proprietary applications, such as web browser [65] or Real-Time Media Flow protocol (an extension to Adobe Flash plug-in), provide the benefits for a limited number of application scenarios, whereas HIP, MIP and Stream Control Transport Protocol (SCTP) and their extensions are applicable to all kinds of Internet services, similarly to MPTCP.

Next, MPTCP is compared to its most potential rivals. These include TCP as the dominant incumbent protocol, MIP and SCTP due to their status as well-defined standards track protocols.

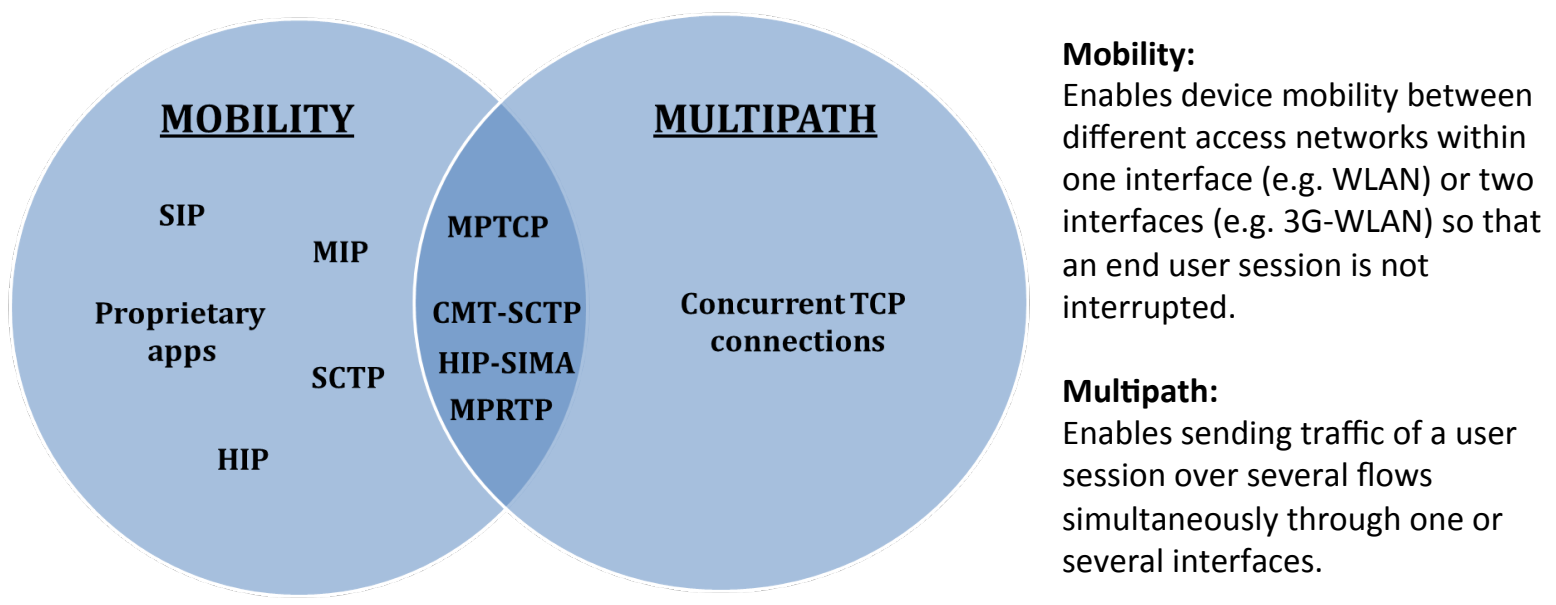

Figure 6. Substitutes of MPTCP (excluding single-path TCP)

Comparison to substitutes: In the literature, MPTCP is compared mostly with TCP, which is the dominant incumbent protocol. From the deployment perspective, MPTCP is a backwards-compatible alternative to TCP with fail-over support. The measurements of Raiciu et al. [66] suggest that MPTCP over 3G and WLAN can achieve almost the double of the goodput of regular TCP using only one of these two. However, the connection setup latency is worse for MPTCP due to the generation of a verification key to link the subflows together. Consequently, MPTCP performs worse for short connections (less than $30 \mathrm{kB}$ ). Since MPTCP is supposed to increase the bandwidth, i.e., decrease the download times the usage of the multiple interfaces has will probably have an increasing effect on the energy consumption of the device. The degree of energy increase depends on various factors such as the type of the wireless interfaces and the functionalities (mobility, multipath) in use [67].

MIP [68] has a status of a well-defined standards track protocol. It supports mobility over a single interface but requires multihoming towards several ISPs. Essentially, MIP improves resiliency of the connection when the end user changes between different access networks. 
The biggest drawback of MIP, besides the lack of deployment despite being standardized already 15 years ago, is that MIP has no access to the information needed to perform optimally in make-before-break mobility events [69]. Additionally, the lack of support for multipath transfer limits its usage in use cases where throughput maximization is desirable [69].

SCTP [70] is more relevant substitute than MIP, given its inherent support for multiaddressed hosts and fail-over. Several SCTP extensions (e.g., [71,72]), including a standardization effort in the IETF [73], enable hosts to use multiple paths at the same time. Although implemented in multiple operating systems, SCTP is still not widely used besides specific applications. SCTP suffers from deployability issues, given it will not get through NATs and firewalls by default [74]. Attempts to encapsulate SCTP in UDP are not widely used either. Furthermore, applications need to explicitly request SCTP, which mandates application changes. Contrary to SCTP, MPTCP has been designed based on a need for something that retains network and application compatibility.

SWOT analysis: Table 2 summarizes the strengths, weaknesses, opportunities and threats of MPTCP from the perspective of successful deployment. The table has been constructed with the help of PEST analysis to ensure that all the relevant domains have been taken into account.

Table 2. SWOT analysis for MPTCP deployment

\section{Strengths}

- Backwards-compatibility with TCP

- NAT traversal capability

- As a transport-layer solution, MPTCP is applicable to wide range of applications

\section{Opportunities}

- Number of multihoming capable devices is increasing

- Emerging applications have higher availability requirements

- Battery life-times are increasing

\section{Weaknesses}

- Changes to TCP/IP stack require OS kernel changes

- Intended RFC status is experimental instead of standards track

- Multipath communication may drain the battery of mobile devices

\section{Threats}

- Single-path bandwidth suffices

- Substitutes are earlier in the market

- Internet connectivity prices remain high

- Router vendors resist MPTCP due to its impact to traffic engineering

\subsection{Feasibility analysis}

The feasibility analysis synthesizes and summarizes the results of Levä et al. [10], Kostopoulos et al. [11], Warma et al. [12], Suomi et al. [13][14] and Sonntag et al. [19] to analyze the costs and benefits of MPTCP deployment for each relevant stakeholder. For more elaborated feasibility analysis, please refer to those papers. The analysis is conducted in 
comparison with the current situation, meaning that the costs and benefits are presented relative to regular TCP. Also, the impact of network effects to the costs and benefits is taken into account. Based on the presented feasibility analysis, the relevant deployment challenges are listed in the end of this section.

Costs and benefits for OS vendors: The costs for OS vendors incur from implementing and maintaining MPTCP in their OSes, and providing support for end users in case they face problems with MPTCP. Monetary costs for implementing MPTCP have not been estimated but implementing MPTCP in an OS kernel is quite burdensome, as described in the papers of Barre et al. [75] and Raiciu et al. [66]. Modifying the TCP/IP stack that is a critical component in OSes may also be perceived too risky. The maintenance and support costs during the lifetime of an OS, which are often larger than the implementation costs, create another concern that limits the implementation of new protocols. As a consequence, OS vendors need to perceive substantial benefits from MPTCP deployment. On the one hand, demand for MPTCP from end users or application developers could help to sell the new OS version directly or through increased supply of interesting applications for the OS. On the other hand, MPTCP could create new business opportunities, for example, by enhancing existing or enabling new OS vendor provided services.

Costs and benefits for end users (consumers and content providers): End users face costs both from acquiring multihoming support and from purchasing and installing MPTCP software to their devices. Fulfilling the end users' multihoming prerequisite is likely to be motivated mainly by other reasons than MPTCP, such as the consumers' need for ubiquitous access or the content providers' desire to have backup connections. If this were the case, MPTCP would be seen as adding value to multihoming, not a reason for multihoming costs. Additionally, many content providers and mobile end users are already multihomed. The cost involved with the adoption of MPTCP software is measured partly in terms of money and partly in terms of time and effort. The costs depend heavily on the adoption model. Content providers typically make a conscious adoption decision with clearly defined costs for purchasing and installing the MPTCP software. Consumers, for one, often adopt MPTCP unintentionally alongside a new OS version, either through an OS update, OS version upgrade or new device acquisition, the cost of which is not associated with MPTCP adoption. While the perceived cost is low in unintentional adoption, the penetration of MPTCP capability to consumers increases slowly with long OS update cycles.

Consumers and content providers see the benefits of MPTCP differently. For consumers, MPTCP improves the perceived service quality. Support for mobility allows session continuity and higher throughput translates into savings in time. However, MPTCP may be more appealing when used over one access link at a time compared to running several access connections in parallel, because the additional energy consumption due to simultaneous usage of multiple radio interfaces may outperform the increase in throughput. For content providers, the benefits are indirect and translate into revenue in case the better service quality increases end users' willingness to pay. The higher resilience enabled by session continuity hides the connection problems from end users and the higher throughput (and related time saving for 
end users) means potential for more sales. Finally, realization of the benefits of MPTCP depends heavily on the availability of communication partners with MPTCP support.

Costs and benefits for ISPs: In the analyzed value network configuration, ISPs do not face direct deployment costs. However, the increased competition among ISPs due to higher visibility of throughput and network performance enabled by MPTCP, and the reduction in provider lock-in due to increased multihoming, may be seen as costs by ISPs. However, innovative ISPs should see this as an opportunity to answer to the increased demand for connectivity and to attract more customers. MPTCP may also allow ISPs to run closer to capacity, since spikes on load can be spread across links more effectively. Additionally, MPTCP may be able to mask occasional, unexpected outages, and thus decrease the number of support calls that present a major cost for ISPs. The behavior of ISPs is hard to predict and becomes visible only after the MPTCP traffic starts to flow on the Internet. The biggest threat caused by ISPs is that their middleboxes would block MPTCP traffic either purposely or accidentally. However, MPTCP has been designed as middlebox-friendly as possible and thus this is not seen as a relevant deployment challenge.

Deployment challenges of MPTCP: In conclusion, the relevant deployment challenges of MPTCP in the chosen use case include:

1. Network performance is sufficient for the end users even without the deployment of MPTCP.

2. OS vendors perceive the costs to implement and maintain MPTCP code and provide support for end users larger than the revenue it brings as an additional OS feature.

3. Chicken-egg problem: Content providers do not start providing MPTCP services because there are no consumers with MPTCP support, while consumers do not adopt MPTCP due to lack of services using it.

\subsection{Solution analysis}

Below is a list of potential solutions for the deployment challenges. Many of them are listed in the paper of Kostopoulos et al. [11].

Open source implementation of MPTCP: Implementing MPTCP in an open source OS, such as Linux, could be used to create competitive pressure and demonstrate the benefits and feasibility of MPTCP for the vendors of commercial operating systems.

Application implementation: The participation of the OS vendors would not be required if MPTCP would be implemented directly into applications or as application-layer middleware. An attractive application benefiting from MPTCP could also accelerate consumer adoption by increasing the importance of intentional adoption.

Lobbying: Due to the large role of unintentional adoption among consumers, and the related larger network effect of consumer adoption to content providers than vice versa, incentivizing the OS vendors to implement MPTCP is crucial. Lobbying could be one solution to increase the knowledge of MPTCP and its benefits among OS vendors. 
Both ends in one hand: A simple way to solve the chicken-egg problem between the consumers and content providers is to change the value network so that a single stakeholder (e.g., Apple, Nokia, Google) controls the operating systems on both mobile devices and on the content servers. The feasibility of this kind of value network is further analyzed in the paper of Warma et al. [12].

Proxy implementation: If the diffusion of MPTCP to consumer devices is too slow or acquiring multihoming infeasible for consumers, MPTCP could be implemented in an ISPprovided proxy. This change in both technical architecture and value network would increase the role of ISPs and introduce new incentives to them.

\section{Discussion}

In this paper, a framework for studying the techno-economic feasibility of Internet protocols has been constructed and its application has been demonstrated with a protocol case. The appliers of the framework should keep a few issues in mind about its opportunities and limitations. Firstly, the framework is based on a limited number of protocol case studies conducted by the authors, which calls for further validation by wider audience. An initial validation has been conducted by university students who applied the framework to six protocols. The received feedback was positive and allowed the authors to refine the framework. Secondly, application of the framework does not guarantee successful deployment of a protocol. However, it can increase the likelihood of adoption through the identification of deployment challenges in the early phase of protocol development. Thirdly, due to its generic nature, the framework can potentially be used to analyze also other networked innovations than IETF protocols, such as proprietary protocols or 3GPP/IEEE standards. This extension, however, remains a topic for future research.

Two target groups should be interested in using the framework. Firstly, improved understanding of the stakeholders' incentives helps protocol developers to avoid design decisions leading to non-deployment and take strategic actions to facilitate the deployment. Secondly, potential adopters can make more informed adoption decisions if they understand, whether the other involved stakeholders are interested in doing their part or not. Organizations belonging to the mentioned target groups participate in the IETF standardization, and therefore, researchers and engineers in the IETF could be the first potential users of the framework. Some of the working groups in the IETF have already been studying the use cases and deployment considerations of certain protocols, but this framework provides a more holistic and systematic baseline for such studies.

Even though the framework has been targeted for people with technical background and the authors have tried to avoid complex economic terminology, the full understanding of the framework and tools may require some experience on economics, business and Internet market dynamics or support from people familiar with these topics. Typically, a more elaborate analysis can be conducted if the developer of the analyzed protocol joins the feasibility study with the support of people with business-oriented background. Research projects should encourage this collaboration by avoiding separation of technical development and socio-economic research into different work packages. Sometimes the collaboration may 
be challenging due to the different and potentially conflicting terminology and concepts. Therefore, the techno-economic feasibility studies also require people with multi-disciplinary expertise to fill the gap between engineers and business managers.

The flexibility of the framework allows variation in its application. For example, the emphasis of the different steps depends on the objectives of its user. Academic researchers may be more interested in an elaborate quantitative analysis of a specific problem in the feasibility analysis step whereas the practitioners may find more value in the initial steps for comparing the different use case, technical architecture and value network alternatives and for analyzing the deployment environment. Furthermore, a clear separation between the actions in each step has been made for clarity purposes. This is forced to some extent, and in practice, the analyses of different steps often overlap. For example, while considering the alternatives for the technical architecture, the modeler often intuitively prospects the related value network, since these two are highly inter-linked. Similarly, the comparison of substitutes is often made unconsciously from the end user perspective. The integration of different steps must not be avoided and is even recommended if it improves the results of the analysis.

In order to improve the accuracy of the results, the application of the framework is intended to be repeated as the protocol design, deployment environment, and other issues regarding deployment change. Nevertheless, the feasibility analysis may fail to predict the outcome of the protocol deployment. This might be because some companies may act irrationally and continue advancing the protocol in the desire for selling more products and in order to show their technical capabilities, although demand for the protocol would be low. In addition, sometimes the availability of technologies just creates demand, which is hard to understand beforehand. Consider, for example, the famous quote of Thomas Watson, the chairman of IBM, in 1943: "I think there is a world market demand for maybe five computers". Therefore, the framework is not intended for predicting the fate of protocols but rather for improving the understanding of their techno-economic feasibility. The framework should yield equally good results as the applier's understanding of the world.

\section{Acknowledgments}

The authors wish to thank Thomas Casey, Heikki Hämmäinen, Kalevi Kilkki, Miika Komu, Oleksiy Mazhelis, and Timo Smura for their constructive comments. The authors also thank the anonymous reviewers for their insightful critique.

The work has been supported by the Internet of Things program of Tivit (Finnish Strategic Centre for Science, Technology and Innovation in the field of ICT), Graduate School in Electronics, Telecommunications and Automation (GETA), and the Future Internet Graduate School (FIGS).

\section{Biographies}

Tapio Levä received his M.Sc. in communications engineering from Helsinki University of Technology (TKK), Finland, in 2009 with major in Networking Technology and minors in Telecommunications Management and Interactive Digital Media. He is currently doing postgraduate studies in the Department of Communications and Networking at Aalto 
University. His research interests include techno-economics of Internet architecture evolution, Internet standards adoption and information-centric networking.

Henna Suomi received her M.Sc. in communications engineering from Helsinki University of Technology (TKK), Finland, in 2009 with major in Telecommunications Management and minor in Networking Technology. After working as a visiting researcher at McGill University she started doing her doctoral studies in the Department of Communications and Networking at Aalto University. Her current research interests include techno-economics of Internet architecture evolution, adoption of multipath protocols and economic effects of multihoming.

\section{References}

[1] J. Arkko, Document Statistics - What is going on in the IETF? Online document, available at: http://www.arkko.com/tools/docstats, accessed January 7, 2013.

[2] M. Handley, Why the Internet only just works, BT Technology Journal, vol. 24, no. 3, 2006, pp. 119-129.

[3] A. Ozment, S.E. Schechter, Bootstrapping the adoption of internet security protocols. Proc. Fifth Workshop on the Economics of Information Security, June 26-28, 2006, Cambridge, England.

[4] J. Marcus, Evolving core capabilities of the Internet. Journal on Telecommunications and High Technology Law, vol. 3, 2004, pp. 121-161.

[5] B. Carpenter, Architectural Principles of the Internet, RFC 1958 (Informational), June 1996.

[6] R. Bush, D. Meyer, Some Internet Architectural Guidelines and Philosophy, RFC 3439 (Informational), December 2002.

[7] D. Thaler, B. Aboba, What Makes For a Successful Protocol? RFC 5218 (Informational), July 2008.

[8] D. Joseph, N. Shetty, J. Chuang, I. Stoica, Modeling the Adoption of New Network Architectures, in Proceedings of International Conference on Emerging Networking Experiments and Technologies (CoNEXT'07), December 10-13, 2007, New York, USA.

[9] S. Sen, Y. Jin, R. Guérin, K. Hosanagar, Modeling the Dynamics of Network Technology Adoption and the Role of Converters, IEEE/ACM Transactions on Networking, vol. 18, no. 6, 2010, pp. 1793-1805.

[10] T. Levä, H. Warma, A. Ford, A. Kostopoulos, B. Heinrich, R. Widera, P. Eardley, Business Aspects of Multipath TCP Adoption. In G. Tselentis et al. (Eds.) Towards the Future Internet, IOS Press, Amsterdam, 2010, pp. 21-30.

[11] A. Kostopoulos, H. Warma, T. Levä, B. Heinrich, A. Ford, L. Eggert, Towards Multipath TCP Adoption: Challenges and Opportunities, in Proceedings of the 6th Euro-NF Conference on Next Generation Internet, June 2-4, 2010, Paris, France.

[12] H. Warma, T. Levä, L. Eggert, H. Hämmäinen, J. Manner, Mobile Internet in Stereo: an End-to-End Scenario, $3^{\text {rd }}$ Workshop on Economic Traffic Management (ETM 2010), September 6, 2010, Amsterdam, the Netherlands.

[13] H. Warma, T. Levä, H. Tripp, A. Ford, A. Kostopoulos, Dynamics of Communication Protocol Diffusion: the Case of Multipath TCP. Netnomics, vol. 12, no. 2, 2011, pp. 133-159.

[14] H. Suomi, K. Kilkki, H. Hämmäinen, Modeling the Value of End-to-End Multipath Protocols. Journal of Universal Computer Science, vol. 18, no. 14, 2012, pp. 2071-2092.

[15] L. Iannone, T. Levä, Modeling the economics of Loc/ID Split for the Future Internet. In G. Tselentis et al. (Eds.) Towards the Future Internet, IOS Press, Amsterdam, 2010, pp. 11-20. 
[16] A. Mäkelä, H. Warma, K. Kilkki, A. Decros, J. Manner, Economic feasibility analysis of seamless multihoming WAN solution, in Proceedings of 7th EURO-NGI Conference on Next Generation Internet (NGI), pp.1-8, June 27-29, 2011, Kaiserslautern, Germany.

[17] T. Levä, M. Komu, A. Keränen, S. Luukkainen, Adoption Barriers of Network-layer Protocols: the Case of Host Identity Protocol, Computer Networks, vol. 57, no. 10, 2013, pp. 2218-2232.

[18] T. Levä, O. Mazhelis, H. Suomi, Comparing the cost-efficiency of CoAP and HTTP in web-of-things applications, Decision Support Systems-Special Issue on Business Applications of Web of Things (2013), (in press).

[19] S. Sonntag, H. Suomi, Techno-economic feasibility of multipath protocols in mobile internet of things applications, Concurrency and Computation: Practice and Experience-Special Issue on Internet of Things (2013), (in press).

[20] E.M. Rogers, Diffusion of Innovations, $5^{\text {th }}$ edition. Free Press, New York, 2003.

[21] F. Davis, Perceived usefulness, perceived ease of use, and user acceptance of information technology. MIS quarterly, vol. 13, no. 3, 1989, pp. 319-340.

[22] M.L. Katz, C. Shapiro, Technology Adoption in the Presence of Network Externalities. Journal of Political Economics, vol. 94, no. 4, 1986, pp. 822-841.

[23] W. Arthur, Competing technologies, increasing returns, and lock-in by historical events, The Economic Journal, vol. 99, no. 394, 1989, pp. 116-131.

[24] B. Briscoe, A. Odlyzko, B. Tilly, Metcalfe's law is wrong - communications networks increase in value as they add members-but by how much? IEEE Spectrum, vol. 43, no. 7, July 2006, pp. 34-39.

[25] F.M. Bass, A New Product Growth Model for Consumer Durables, Management Science, vol. 13, no. 5, 1969, pp. 215-227.

[26] R.G. Fichman, C.F. Kemerer, Adoption of software engineering process innovations: the case of object orientation, Sloan Management Review, vol. 34, 1993, 7-22.

[27] J.H. Saltzer, D. Reed, D.D. Clark, End-to-End Arguments in System Design, ACM Transactions on Computer Systems, vol. 2, no. 4, 1984, pp. 277-288.

[28] D. Clark, J. Wroclawski, K. Sollins, R. Braden, Tussle in Cyberspace: Defining Tomorrow's Internet. IEEE/ACM Transactions on Networking, vol. 13, no. 3, 2005, pp. 462-475.

[29] A. Ford, P. Eardley, B. van Schewick, New Design Principles for the Internet, in Proceedings of International Workshop on the Network of the Future, June 14-18, 2009, Dresden, Germany.

[30] A. Hovav, R. Patnayakuni, D. Schuff, A Model of Internet Standards Adoption: the Case of IPv6. Information Systems Journal, vol. 14, no. 3, 2004, pp. 265-294.

[31] E.U. Bond, M.B. Houston, Barriers to Matching New Technologies and Market Opportunities in Established Firms, Journal of Product Innovation Management, vol. 20, no. 2, 2003, pp. 120-135.

[32] S. Verbrugge, K. Casier, J. Van Ooteghem, B. Lannoo, Practical steps in techno-economic evaluation of network deployment planning, White paper, November 25, 2009.

[33] P. Eardley, M. Kanakakis, A. Kostopoulos, T. Levä, K. Richardson, H. Warma. Deployment and Adoption of Future Internet Protocols, in Future Internet Assembly 2011: Achievements and Technological Promises, Lecture Notes in Computer Science, vol. 6656, Springer 2011, pp. 133-144.

[34] C. Kalogiros, C. Courcoubetis, G.D. Stamoulis, M. Boniface, E. Meyer, M. Waldburger, D. Field, et al., An Approach to Investigating Socio-economic Tussles Arising from Building the Future Internet, in Future Internet Assembly 2011: Achievements and Technological Promises, Lecture Notes in Computer Science, vol. 6656, Springer, 2011 pp. 145-159. 
[35] A. Kostopoulos, I. Papafili, C. Kalogiros, T. Levä, N. Zhang, D. Trossen. A Tussle Analysis for Information-centric Networking architectures, in The Future Internet, Lecture Notes in Computer Science, vol. 7281, Springer, 2012, pp. 6-17.

[36] A. Ford, C. Raiciu, M. Handley, S. Barre, J. Iyengar, Architectural Guidelines for Multipath TCP Development, RFC 6182 (Informational), March 2011.

[37] I.-W. Wu, W.-S. Chen, H.-E. Liao, F.F. Young, A seamless handoff approach of Mobile IP protocol for mobile wireless data networks, IEEE Transactions on Consumer Electronics, vol. 48, no. 2, 2002, pp. 335344.

[38] R. Freeman, Strategic Management: A Stakeholder Approach, Boston: Pitman, 1984.

[39] R. Mitchell, B. Agle, D. Wood, Toward a Theory of Stakeholder Identification and Salience: Defining the Principle of Who and What Really Counts, The Academy of Management Review, vol. 22, no. 4, 1997, pp. 853-886.

[40] T. Casey, T. Smura, A. Sorri, Value Network Configurations in wireless local area access. 9th Conference of Telecommunication, Media and Internet (CTTE), June 7-9, 2010, Ghent, Belgium.

[41] V. Allee, Reconfiguring the Value Network, Journal of Business Strategy, vol. 21, no. 4, 2000, pp. 36-39.

[42] A. Mendelow, Stakeholder mapping, in Proceedings of the 2nd international conference on information systems, 1991, MA, Cambridge.

[43] H. Zhao, Emerging Business Models of the Mobile Internet Market. M.Sc. Thesis, Helsinki University of Technology, 2008.

[44] P. Faratin, Economics of overlay networks: an industrial organization perspective on network economics, in Proceedings of NetEcon $+I B C$, June 11, 2007, San Diego, California.

[45] N. Zhang, T. Levä, H. Hämmäinen, Value Networks and Two-Sided Markets of Internet Content Delivery, Telecommunications Policy, Available online 16 May 2013, ISSN 0308-5961, http://dx.doi.org/10.1016/j.telpol.2013.03.004

[46] A.T. Campbell, J. Gomez, S. Kim, C.-Y. Wan, Comparison of IP micromobility protocols, IEEE Wireless Communications, vol. 9, no. 1, 2002, pp. 72-82.

[47] F. Aguilar, Scanning the business environment, New York: Macmillan, 1967.

[48] D.W. Pickton, S. Wright, What's swot in strategic analysis?, Strategic Change, vol. 7, no. 2, 1998, pp. 101-109.

[49] P. Schoemaker, Scenario planning: A tool for strategic thinking, Sloan Management Review, vol. 36, 1995, pp. $25-40$.

[50] B. Karlson, A. Bria, P. Lönnqvist, C. Norlin, J. Lind, Wireless Foresight: Scenarios of the mobile world in 2015, Chichester, UK: Wiley, 2003.

[51] M. Heikkinen, M. Matuszewski, H. Hämmäinen, Scenario planning for emerging mobile services decision making: mobile Peer-to-Peer Session Initiation Protocol case study, International Journal on Information and Decision Sciences, vol. 1, no. 1, 2008, pp. 26-43.

[52] T. Smura, A. Sorri, Future Scenarios for Local Area Access: Industry Structure and Access Fragmentation, in Proceedings of the Eighth International Conference on Mobile Business (ICMB 2009), June 27-28, 2009, Dalian, China.

[53] T. Levä, K. Kilkki, H. Hämmäinen, Scenario Analysis on Future Internet, in Proceedings of the First International Conference on Evolving Internet (INTERNET 2009), August 23-29, 2009, Cannes/La Bocca, France. 
[54] L. Tang, Q. Chen, B. Zhang, Y. Li, X. Zheng, Load balancing model based on Stackelberg game for multihoming in heterogeneous radio access networks, in Proceedings of the 2008 International Conference on Advanced Infocomm Technology - ICAIT '08, July 28-31, 2008, Da Meisha, Shenzhen, China.

[55] T. Smura, Techno-economic modeling of wireless network and industry architectures, PhD dissertation, Aalto University, School of Electrical Engineering, 2012.

[56] T. Luehrman, Using APV: A Better Tools for Valuating Operations, Harvard Business Review, May-June, 1997, pp 145-154.

[57] L. Ellram, A Framework for Total Cost of Ownership, The International Journal of Logistics Management, vol. 4, no. 2, 1993, pp. $49-60$.

[58] D.S. Shepard, M.S. Thompson, First Principles of Cost-Effectiveness Analysis in Health, Public Health Reports, vol. 94, no. 6, 1997, pp. 535-543.

[59] J. Sterman, Business Dynamics: Systems Thinking and Modeling for a Complex World, McGrawHill/Irwin, 2000.

[60] J.H. Thun, A. Grössler, P.M. Milling, The diffusion of goods considering network externalities: a system dynamics-based approach, in Proceedings of the 18th International Conference of the System Dynamics Society, August 8, 2000, Bergen, Norway.

[61] A. Kelic, Networking technology adoption: System dynamics modeling of fiber-to-the- home, PhD Thesis, Massachusetts Institute of Technology, 2005.

[62] C. Kalogiros, M. Bagnulo, A. Kostopoulos, Understanding Incentives for Prefix Aggregation in BGP, in Proceedings of ACM CoNEXT, ReArch'09 - Re-Architecting the Internet, December 1, 2009, Rome, Italy.

[63] A. Ford, C. Raiciu, M. Handley, O. Bonaventure, TCP Extensions for Multipath Operation with Multiple Addresses, RFC 6824 (Experimental), January 2013.

[64] M. Sharf, A. Ford, M., Multipath TCP (MPTCP) Application Interface Considerations, RFC 6897 (Informational), March 2013.

[65] M. Komu, A Consolidated Namespace for Network Applications, Developers, Administrators and Users, Doctoral dissertation, Aalto University, 2012.

[66] C. Raiciu, C. Paasch, S. Barre, A. Ford, M. Honda, F. Duchene, O. Bonaventure, M. Handley, How hard can it be? Designing and implementing a deployable multipath TCP, in Proceedings of the 9th USENIX conference on Networked Systems Design and Implementation (NSDI'12), April 25-27, 2012, San Jose, CA.

[67] J.K. Nurminen, Parallel Connections and their Effect on the Battery Consumption of a mobile phone, in Proceedings of the 7th IEEE Consumer Communications \& Networking Conference, Las Vegas, Nevada, 2010.

[68] C. Perkins, IP Mobility Support for IPv4, Revised, RFC 5944 (Proposed standard), November 2010.

[69] C. Raiciu, D. Niculescu, M. Bagnulo, M. Handley, Opportunistic mobility with multipath TCP, in Proceedings of the sixth international workshop on MobiArch (MobiArch '11), June 28, 2011, Washington, D.C.

[70] R. Stewart, Stream Control Transmission Protocol, RFC 4960 (Proposed standard), September 2007.

[71] J. Iyengar, P.D. Amer, R.R. Stewart, Concurrent multipath transfer using SCTP multihoming over independent end-to-end paths, IEEE/ACM Transactions on Networking, vol. 14, no. 5, 2006, pp. 951-964.

[72] J. Liao, J. Wang, X. Zhu, cmpSCTP: An extension of SCTP to support concurrent multi-path transfer, in Proceedings of the IEEE International Conference on Communications (ICC'08), May 19-23, 2008, Beijing, China. 
[73] P. Amer, M. Becke, T. Dreibholz, N. Ekiz, J. Iyengar, P. Natarajan, R. Stewart, M. Tuexen, Load Sharing for the Stream Control Transmission Protocol, Internet-draft, work in progress.

[74] S. Hätönen, A. Nyrhinen, L. Eggert, S. Strowes, P. Sarolahti, M. Kojo: An experimental study of home gateway characteristics, Proceedings of the 10th ACM SIGCOMM conference on Internet measurements (IMC), 2010.

[75] S. Barre, C. Paasch, O. Bonaventure, MultiPath TCP: From Theory to Practice, in Proceedings of the 10th International IFIP TC 6 Networking Conference, May 9-13, 2011, Valencia, Spain. 